\title{
CANOMAD : A multi-faceted disease
}

Júlia Machado Rickli 1, Gabriela Pomaleski 1, Marcus Vinícius Magno Gonçalves. MD PhD 2

1. Medical student - Department of Medicine, University of the Region of Joinville (UNIVILLE), Brazil

2. Professor of Neurology - Department of Medicine, University of the Region of Joinville (UNIVILLE), Brazil

\section{ABSTRACT}

Objective: Elucidate the main clinical aspects of the CANOMAD spectrum. Methods: Bibliographical review trough databases (PubMed, Google Scholar, Orphanet, Oxford Academic) of articles from 1985 (1) to 2019 and later selection of the most applicable of the above, in order to construct a non-systematic review. Conclusion: CANOMAD is a chronic-ataxic autoimmune neuropathy associated with IgM monoclonal gammopathy. The correct diagnosis of this rare and multi-faceted disease will help optimal treatment.

Keywords: chronic-ataxic neuropathy; anti-

disialosyl; IgM monoclonal gammopathy; CANOMAD

\section{INTRODUCTION:}

CANOMAD is a rare syndrome that belong to the group of peripheral neuropathies associated with IgM monoclonal gammopathy (2). This acronym was first used in 1996 to describe the clinical features of the disease, which include: chronic sensory ataxic neuropathy, ophtalmoplegia, M-protein with cold-agglutinin activity and anti-disialosyl antibodies (3). 
The syndrome's physiopathology is not fully understood, but, according to the available researches, it will be expressed in patients whose serum contains IgM antibodies to disialylated gangliosides, mostly, with cold agglutinins evidenced as well (4). The high titles of antigangliosides will react against GDIb, GD3, GT1b e GQ1b, nerve cells elements, in the NeuNAc(alfa2-8) NeuNAc(alfa2-3) terminal epitope. This antibody-epitope binding occurring in sundry peripheral nerves structures, such as ganglion neurons of the dorsal root, peripheral axons and myelin, nodes of Ranvier, muscle spindles and motor nerve terminals will lead to neural involvement (5).

There are also presentations of cold agglutinin with anti-Pr specificity, which about half of patients with CANOMAD have, and it is associated to red cell activity of anti-Prl or anti-Pr2. (4) IgM paraprotein with anti-Pr2 cold agglutinin activity may play identifying the sialic-acid epitopes presents of disialylated gangliosides. Also, the specificity of anti-Pr IgM may be one more determinant factor of the binding to myelin, thus the affection in CANOMAD (6). This paraprotein also acts a play in the pathogenic function in peripheral nerve demyelination. (7) The worsening in cold weather of the patient's neurological symptoms may be justified by the anti-Pr cold agglutinin, as the cause of cold agglutinin disease. (6)

This disease mainly affects male patients with mean onset at 5th decade of life. The clinical presentation of said neuropathy is heterogeneous and the symptoms can appear in relapses. (8) The patients typically manifest with proprioceptive ataxia, ophtalmoplegia and areflexia with relative preservation of motor function. In some cases, have cranial nerve involvement with ocular, sensory or bulbar manifestations (2). Motor weakness mainly affects oculomotor and bulbar muscles, therefore, CANOMAD is a chronic reminiscent of Miller Fisher syndrome. (8) 
Sorologically, CANOMAD is characterized by the presence of IgM monoclonal gammopathy and antibodies against gangliosides with disialosyl groups, including GD3, GT1b, GD1b and GQ1b that share a terminal residue in the glycide structure named as the NeuAc(a23) Gal epitope. (9)

\section{METHOD:}

This nonsystematic review was conducted through articles review in PubMed, Google Scholar, Orphanet, Oxford Academic, databases, applying key words as "CANOMAD", "IgM paraprotein", "chronic ataxic neuropathy", "ophtalmoplegia", "cold agglutinins", "anti-gangliosides", with and without the Boolean Operator "AND" between each pair of them. There were no exclusion factors regarding to idiom nor publication date. The analysis comprised since the pioneer study, in UK, 1996 by Willison and colleagues until the most recent, published in 2018.

The present article construction aimed to elaborate a clear approach synthetizing the available information about the CANOMAD syndrome, it's physiopathology, clinical and serological manifestation as well as the disease's diagnosis and treatment based on the previous literature.

\section{RESULTS:}

All articles accrued from the searches described, that were consistent with the proposed theme, were selected in order to secure a most reliable literature review. Naturally, those whose information about the matter was more valid, according to the physiopathology, clinical manifestation, diagnosis and treatment of the CANOMAD syndrome, were included in this non-systematic review.

\section{DISCUSSION:}


It is estimated that in $5-10 \%$ of the neuropathies, simultaneous serum paraprotein may occur. This justifies the matter of approaching the concurrent paraproteinemia and neuropathy, a complex and heterogeneous entity, in which CANOMAD syndrome is among, being one of the phenotypes, although rare, associated to monoclonal gammopathy of undetermined significance (MGUS) (10).

CANOMAD is an acronym for the corresponding chronic ataxic neuropathy, ophthalmoplegia, IgM-paraprotein, cold agglutinins and disialosyl antibodies. It portrays a clinical-serological syndrome defined by some features as the presence of paraprotein IgM, cold agglutinins and disialosyl antibodies damaging neurological tissue, causing sensory ataxia, motor weakness, including ocular motor skills and bulbar muscles commitment.

The background of CANOMAD disease consists in the presence of IgM antibodies in the serum, that reacts mainly with NeuAc (alfa28) NeuAc (alfa2-3) Gal-configured disialosyl epitopes, which are common to many gangliosides, including GDIb, GD3, GT1b and GQ1b. Gangliosides are glycosphingolipids of the cell membrane, mainly in nerve cells (11).

By GDIb, GD3, GTIb antibodies recognition and binding to the respective oligosaccharide chain in peripheral nerves structures, such as peripheral axons, myelin, dorsal root ganglion neurons, nodes of Ranvier, and also motor nerve terminals and muscle spindles, entailing neuronal damage and further symptoms (4). Thus, gangliosides depletion by IgM antibodies antigangliosides will consequently degenerate sensory neurons per degrade it's nodal axolemma and impairing myelination as inducing demyelination, resulting in the described nervous system affection (10). 
There may also be cold agglutinins in CANOMAD's physiopathology, which include anti-Pr2 antibody nearby the PNS's capillary endothelia, framing an access of the immune mechanisms to PNS myelin through this brain-blood-barrier immune damage (figure 1). Once in this syndrome's picture, histologically, the tissue presentation may show either axonal degeneration or demyelination. (1)

Clinically, CANOMAD's onset is predominantly chronic, few are acute or subacute (12) and it affects mostly men around the age of 55. When in woman, this start is inclined to happen earlier, in the fourth decade (4). Primarily, it comprises a chronic neuropathy with sensorial impairment and ataxic gait disorder accompanied by relatively preserved motor function in the limbs and areflexia or hyporeflexia in most cases. Sensory ataxia is found in all cases. The motor disturbances alter mainly oculomotor and bulbar muscles and may be either fixes symptoms or relapsing-remitting features. Also, cranial nerve sensory and motor involvement, fixed or relapsing-remitting, being the most affected the $\mathrm{CN}$ III, therefrom, the oculomotor commitment; it distinguishes CANOMAD from other paraproteinemic neuropathies (4). Nevertheless, loss of kinesthesia, small fiber sensation, as numbness, vibration sense and proprioception, having relatively preserved muscle strength. Furthermore, acral and perioral paresthesia often featured as well. (4).

Other manifestations are external ophthalmoplegia, dysphagia, dysarthria. Respiratory muscle weakness is rarely observed. Symptoms persistence is widely variable about its duration in years, from 4 years up to 4 decades, but often extended over decades (13)(4). It is reasonable to mention CANOMAD syndrome's course can be wavering over its chronicity (14).

Attesting CANOMAD syndrome is a challenge due to its rarity and miscellaneous symptoms criteria, besides some important differential 
diagnosis like Miller Fisher Syndrome and Guillain-Barré Syndrome, a wide investigation becomes necessary regarding to clinical and laboratory findings.

The diagnosis of CANOMAD requires having the clinical features of motor weakness, sensory ataxia, and in some cases reduced or absent reflexes as well as cranial nerve irregularities. Besides the presence of IgM antibodies against ganglioside with disialosyl groups, specificaly GD3, GT1b, GQ1b and GT1b. Antibody testing was perfomed via enzyme-like immunosorbent assay (ELISA) and the most patients had elevated serum IgM rates. (10) The presence of cold agglutinins varied according to the case. The clinical picture is variable as the antibody responds against desialylated gangliosides (8).

Neuroimaging exams (MRI and CT scan) usually is normal (2) but nerve biopsy indicate demyelination and axonal damage; also, electrophysiology and pathology show signs characteristic of axonal flawed. (11) The grading as severe, mildly deranged or normal is based in the demyelinating, axonal, motor and sensorial features. (4)

Also, trace of nerve enlargement which is consonant with acquired demyelination was detected through neuromuscular ultrasound once performed in CANOMAD affected person. This finding was both qualitatively and quantitatively associated to demyelinating neuropathy in contrast to non polyneuropath patients. Eventually characterizing nerve ultrasound as a suggestion for additional diagnosis test, regarding the requirement of further analysis. $(1,14)$

The nature of phenotype the CANOMAD is chronic, progressive and variable, what makes difficult to evaluate the effect of treatment. $(15,16)$ Due to this and the scarce of case series, case reports and the absence of randomized trials, the treatment guideline is 
limited. (10) However, even if there is no evidence-based treatment, according to a few case reports, the therapy with intravenous immunoglobulin (IVlg) may be able to avoid fluctuations of symptoms in CANOMAD because of the antibody neutralization by IVIg improving nerve function. (17)

Furthermore, the rituximab is considered to be optimal used in antiMAG neuropathies. Yet, as a monoclonal antibody anti-CD20, it is not able to suppress CANOMAD activity, once the paraprotein $\lg M$ is antigen-induced by long-lived CD20 negative plasma cells, which explains the poor response to rituximab treatment. $(17,18)$ Though, owing to the disease's site, rituximab might not be able to reach B Limphocytes in the nervous system due to the large size of the molecule incapable to cross the brain blood barrier. $(13,16)$

\section{CONCLUSION:}

CANOMAD is a chronic-ataxic autoimmune neuropathy associated with IgM monoclonal gammopathy. The correct diagnosis of this rare and multi-faceted disease will help optimal treatment.

\section{BIBLIOGRAPHY:}

1. Ilyas AA, Quarles RH, Dalakas MC, Fishman PH, Brady RO. Monoclonal IgM in a patient with paraproteunemic polyneuropathy binds to gangliosides containing disialosyl groups. Ann Neurology (1985). 18(6):655-9. DOI: 10.1002/ana.410180605

2. Toussirot $E$, Béreau $M$, Sevrin $P$, Tatu L. An unusual association of CANOMAD and rheumatoid arthritis with a long term follow-up. Joint Bone Spine. (2019) DOI:10.1016/j.jbspin.2019.09.009. 
3. Sanvito L, Rajabally Y. Optic Neuropathy associated with CANO MAD: description of 2 cases. Muscle \& nerve. (2011). 44. 4515. DOI: 10.1002/mus.22157.

4. Willison HJ, O'Leary CP, Veitch J, Blumhardt LD, Busby M, et al. The clinical and laboratory features of chronic sensory ataxic ne uropathy with anti-disialosyl IgM antibodies. Brain (2001) 124: 1968-1977.

5. Rojas-Garcia R, Martinez-Lage M, Gallardo E, de Luna N, MartiVilalta J. (2006). A novel antiganglioside specificity against terminal NeuNAc(alfa 2-3)Gal in acute bulbar palsy. Journal of Neuroimmunology, 176(1-2), 219-222.

doi:10.1016/j.jneuroim.2006.04.001

6. Herron B, Willison HJ, Veitch J, Roelcke D, llis LS, Boulton FE. (1994). Monoclonal IgM Cold Agglutinins with Anti-PrldSpecificity in a Patient with Peripheral Neuropathy. Vox Sanguinis, 67(1), 5863. DOI:10.1111/j.1423-0410.1994.tb05039.x

7. Willison HJ, Paterson G, Veitch J, Inglis G, Barnett S. (1993). Peripheral neuropathy associated with monoclonal lgM anti-Pr2 cold agglutinins. Journal of neurology, neurosurgery, and psychiatry, 56(11), 1178-1 183. DOI: 10.1136/jnnp.56.11.1178

8. Delval A, Stojkovic T, Vermersch P. Relapsing sensorimotor neurop athy with ophthalmoplegia, antidisialosyl antibodies, and extram embranous glomerulonephritis. Muscle \& Nerve, (2006).33(2), 274-277. DOI:10.1002/mus.20452

9. Garcia-

Santibanez R, Zaidman C, Sommerville R, Lopate G, Weihl C, Pest ronk $A$, Bucelli R. CANOMAD and other chronic ataxic neuropat hies with disialosyl antibodies (CANDA). Journal of Neurology. (2018). 265. DOI: 10.1007/s00415-018-8853-4.

10. Von K, Ferreira, Marinho R, Castro L, Nigro L, Montero Edna. (2000). Gangliosideos - estudo do colágeno e da resposta 
inflamatória no processo cicatricial. Acta Cirurgica Brasileira. 14. DOI: $10.1590 /$ S0102-86502000000500005.

11. Krenn M, Keir G, Wieshmann U.CANOMAD responding to weekly treatment with intravenous immunoglobulin (IVIg). BMJ case reports. (2014). DOI: 10.1 136/bcr-2013-202545.

12. Arait, Yoshino H, Kusano Y, Yazaki Y, Ohnishi Y, Miyatake T. Ataxi c polyneuropathy and antiPr2 lgMk M proteinemia. Journal of Neurology (1992). 239:147151. DOI: $10.1007 /$ BF00833915.

13. Halpin S, Al-Hussayni S, Hasan S, Busby M, Buccoliero R. A case of CANOMAD with Review of the Literature. Brain Disorders \& Therapy (2015). 4: 3. DOI: 10.4172/2168-975X.1000166

14. Genetic and Rare Diseases Information Center (GARD).

15. Löscher WN, Woertz A, Wallnöfer M, Wanschitz JV, Luef, G. Successful treatment of CANOMAD with IVIg and rituximab. J ournal of Neurology, (2013). 260(4), 11681170. DOI:10.1007/s00415-013-6867-5.

16. Mckelvie PA et al. CANOMAD: report of a case with a 40year history and autopsy. Is this a sensory ganglionopathy with ne uromuscular junction blockade? Muscle \& Nerve, (2013) 48 (4), 599-603. DOI:10.1002/muc.23897

17. Siddiqui K, Cahalane E, Keogan M, Hardiman O. Chronic ataxic neuropathy with cold agglutinins: Atypical phenotype and respo nse to anti-CD20 antibodies. Neurology, (2003). 61(9), 13071308. DOI:10.1212/wnl.61.9.1307

18. Rojas-Garcia R, Gallardo E, De Andres I, de Luna N, Juarez C, Sanchez P. Chronic neuropathy with IgM anti-ganglioside antibodies: Lack of long term response to rituximab. Neurology, 61 (12) 1814-1816. DOI: 10.1212/01/wnl.0000098996.02934.86 